\title{
Crecimiento de la mojarra tilapia variedad Stirling durante la etapa de desarrollo, bajo dos dietas alimenticias
}

Tilapia growth crappie variety Stirling during the development stage, under two diets

Otoniel-Hernández Porfirio ${ }^{1}$, Santos-Ángel Bruno Martínez ${ }^{1}$, Guadalupe-Castillo Capitán ${ }^{1 凶}$, Maximino-Zito Romero Figueroa ${ }^{1}$, María-Gisela Velázquez Silvestre ${ }^{1}$

\author{
${ }^{1}$ Facultad de Ingeniería en Sistemas de Producción Agropecuaria. Universidad Veracruzana, Km 4.5 \\ Carretera Costera del Golfo Acayucan-Catemaco, Acayucan, Veracruz. CP. 96000. Tel y Fax (924)24- \\ 7-91-22. \\ ${ }^{\otimes}$ Autor para correspondencia: gcastillo@uv.mx
}

Recibido: 25/01/2016

Aceptado: 28/06/2016

\section{RESUMEN}

Se evaluó el crecimiento y el peso de tilapia variedad Stirling durante la etapa de desarrollo, a partir del suministro de dos dietas alimenticias: alimento comercial y dieta a base de harina de carne. El trabajo se realizó bajo un sistema de producción semi-intensivo en dos estanques de concreto con capacidad de $24 \mathrm{~m}^{3}(3 \times 8 \times 1)$, bajo una densidad experimental de 600 peces/estanque y durante tres meses (noviembre, diciembre y enero). Los peces se alimentaron tomando en cuenta su biomasa corporal. Los datos se analizaron con un diseño factorial completamente al azar y 60 repeticiones por cada tratamiento. Los resultados mostraron diferencias significativas para ambas dietas, la ganancia de peso fue mayor con el concentrado comercial (49.65g) que con la dieta elaborada (25.62g). La dieta comercial permite un crecimiento significativo para la Tilapia. La dieta a base de harina de carne minimiza costos de producción, Los resultados obtenidos indican que la dieta a base de harina de carne presenta una diferencia en costo de $66.36 \%$. Si se requiere obtener tilapia de un peso comercial, $>350 \mathrm{~g}$ en poco tiempo, la mejor opción es alimentar con el concentrado comercial, los costos de alimentación se recupera con las ventas. La alimentación a base de harina de carne, es para un productor de autoconsumo de bajos recursos económicos, de mercado local, ventas en menudeo y con más tiempo para la engorda de la tilapia.

Palabras clave: Concentrado, harina de sangre, biomasa, producción. 


\begin{abstract}
The growth of tilapia variety Stirling was evaluated during the development stage, from supply of two diets: commercial food and diet based on meat meal. The work was done under a system of semiintensive production on two concrete ponds with a capacity of $24 \mathrm{~m}^{3}(3 \times 8 \times 1)$, under an experimental density of 600 fish / pond and for three months (November, December and January). The fishes were fed their body taking into account biomass. Data were analyzed with a factorial completely randomized design with 60 repetitions per treatment. The results showed significant differences for both diets, weight gain was higher with the commercial concentrate $(49.65 \mathrm{~g})$ with the elaborate diet $(25.62 \mathrm{~g})$. The commercial diet allows significant growth for Tilapia. The diet of meat meal minimizes production costs, The results indicate that the diet of meat meal has a difference in cost of $66.36 \%$. If required to obtain a commercial weight tilapia,> $350 \mathrm{~g}$ in a short time, the best option is the commercial concentrate feed, feed costs recovers sales. The diet based on meat meal is an alternative to a producer of low-income subsistence, with sales to the local market and with more time feeds to grow tilapia.
\end{abstract}

Keywords: Growth, meat meal, biomass, subsistence

\title{
INTRODUCCIÓN
}

La acuacultura tiene como estrategia principal, la producción de alimentos con alto valor proteico y de bajo costo. Actualmente la rentabilidad de los sistemas acuícolas es afectada por los costos de producción. Se estima que más del $50 \%$ de estos van dirigidos a la alimentación (Shirai, 2006). La tilapia debido a su fácil adaptación a los distintos sistemas de cultivos en los que se ha desarrollado en cautiverio ha permitido que se realicen diferentes estudios que permitan manejar estrategias para minimizar los costos de producción del sistema. El éxito de la acuacultura está vinculado a la reducción de las importaciones y a la evaluación de dietas con mayor proporción de proteína de alto valor proteico (USDA, 2005). Es importante cubrir los requerimientos nutritivos de los individuos de los sistemas acuícolas con recursos naturales, ya que los precios de los alimentos proteicos a nivel mundial se han encarecido (FAO, 2007).
En la acuacultura, los costos de la alimentación están relacionados con el porcentaje de proteína (elemento nutricional más caro de las dietas) que lo constituyen, de ahí la necesidad de generar dietas como estrategia fundamental, en los sistemas intensivos y semi-intensivos, que permitan la disminución de los costos de producción, pero que al mismo tiempo permitan productos de calidad (Shirai, 2006)

Los insumos agroindustriales mezclados funcionan como alternativa para la alimentación de la tilapia, que a la vez, son productos no reutilizables por las industrias, tienen bajo costo en la adquisición (Salas, 2014). Sin embargo, estos ingredientes deben satisfacer los requisitos de calidad nutricional, seguridad e inocuidad de los alimentos, rentabilidad y bienestar animal (Souza, 2012). El principal ingrediente utilizado en la dieta 
para peces, es la harina de pescado debido a su valor nutritivo y palatabilidad, sin embargo, a nivel mundial el costo es elevado. Debido a la importancia que tienen los productos y subproductos que se utilizan en la alimentación de los peces, el presente trabajo expone una dieta alternativa, a partir de la harina de carne, para el crecimiento de la tilapia durante la etapa de desarrollo.

\section{MATERIALES Y MÉTODOS}

El estudio se realizó en el módulo acuícola de FISPA-UV campus Acayucan, Ver., ubicado a $18^{\circ} 00^{\prime} 10.45^{\prime}, \quad \mathrm{N}$ y 9455'38.21', O, a una altura de $105 \mathrm{msnm}$. Su clima es cálido húmedo con una temperatura promedio de $27^{\circ} \mathrm{C}$ (INEGI, 2010). Se utilizaron dos estanques de concreto con capacidad de 24 $m^{3}(3 \times 8 \times 1)$ bajo la densidad experimental de 600 peces/estanque. El diseño experimental fue completamente al azar, con dos tratamientos y 60 repeticiones. Se utilizaron 1200 individuos, separados en lotes de 600 juveniles, se alimentaron con dos tipos de alimento: uno comercial y una ración balanceada. Los juveniles del tratamiento "T1" se alimentaron con el concentrado comercial y el tratamiento "T2" se alimentó con la ración balanceada (harina de carne, maíz molido, aceite de rosticería, sal mineral y melaza). $\mathrm{El}$ experimento inició con peces en la etapa de juveniles con un peso promedio de 3.75 gramos y $5.82 \mathrm{~cm}$, y se realizaron muestreos cada 14 días, para medir el incremento. Para la determinación de la talla se utilizó una regla graduada de $30 \mathrm{~cm}$ y para el peso una balanza digital con capacidad de $5 \mathrm{~kg}$. Se realizó un

análisis de varianza (ANOVA) y posteriormente una prueba de medias por el método Tukey $(\mathrm{p}<0.05)$.

\section{RESULTADOS Y DISCUSIÓN}

El peso promedio fue similar para las dos dietas, durante los primeros tres muestreos, a partir del cuatro muestreo se observa una diferencia en los promedios, teniendo mayor promedio los individuos del tratamiento: ración comercial (cuadro 1), probablemente por la falta de proteína en la dieta elaborada. Esto coincide con los resultados obtenidos por Auró et. al., 2003, en el cultivo de peces de agua dulce alimentados con cerdaza ensilada y empastillada donde se comparan en los dos primeros grupos tres tasas de alimentación (2, 3 y $5 \%$ ) contra dos grupos testigos alimentados con una dieta balanceada, existiendo diferencias significativas a favor del grupo control. Los requerimientos de proteína para la tilapia en la etapa juvenil es de $45 \%$ a $35 \%$ PC con individuos de un peso de 5 a $50 \mathrm{~g}$, con $5 \%$ como máximo de energía (Kcal) y 30\% como máximo de carbohidratos (Meyer et. al., 2007 y Moreno et. al., 2000). 


\begin{tabular}{|c|c|c|}
\hline Fechas & Concentrado comercial & Dieta elaborada \\
\hline $08 / 10 / 2014$ & 3.78 & 3.73 \\
\hline $22 / 10 / 2014$ & 7.82 & 7.98 \\
\hline $05 / 11 / 2014$ & 13.42 & 13.03 \\
\hline $19 / 11 / 2014$ & 17.57 & 15.50 \\
\hline $04 / 12 / 2014$ & 21.73 & 21.00 \\
\hline $19 / 12 / 2014$ & 35.31 & 23.63 \\
\hline $03 / 01 / 2015$ & 53.43 & 29.35 \\
\hline
\end{tabular}

La mejor ganancia de peso al final del experimento se obtuvo con el concentrado comercial (cuadro 2). Esto coincide con lo reportado por: Pérez (2007) y Meyer 2007), quienes mencionan que los alimentos balanceados tienen mejores resultados para los productores de tilapia, que esperan obtener un producto con una talla comercial mayor a los $350 \mathrm{~g}$ en periodos cortos (siete - ocho meses), no así para acuacultores que utilizan dietas con productos agroindustriales, las que requieren mayor cantidad de alimento por pez y mayor tiempo para lograr una talla comercial.

Cuadro 2. Ganancia de peso (g) de juveniles de tilapia, durante la etapa de desarrollo, bajo dos dietas alimenticias.

\begin{tabular}{lrr} 
番 & $\begin{array}{c}\text { Concentrado } \\
\text { comercial }\end{array}$ & \multicolumn{1}{c}{$\begin{array}{c}\text { Dieta } \\
\text { elaborada }\end{array}$} \\
\hline Peso inicial & 3.78 & 3.73 \\
Peso final & 53.43 & 29.35 \\
Ganancia de peso & 49.65 & 25.62 \\
\hline
\end{tabular}

La talla promedio en los primeros dos meses, no presentó diferencia significativa en los dos tratamientos. A partir del tercer mes, la mayor ganancia en talla se obtuvo con el tratamiento concentrado comercial. En la ganancia de talla de la tilapia durante el experimento, el tratamiento concentrado comercial, reveló una diferencia de 2.26 con respecto a la dieta elaborada (figura 2 y cuadro 3 ). 
Cuadro 3. Ganancia de Talla (cm) de juveniles de tilapia, durante la etapa de desarrollo, bajo dos dietas alimenticias.

\begin{tabular}{lcc|} 
& $\begin{array}{c}\text { Concentrado } \\
\text { comercial }\end{array}$ & $\begin{array}{c}\text { Dieta } \\
\text { elaborada }\end{array}$ \\
\hline Talla inicial & 5.95 & 5.69 \\
\hline Talla final & 14.34 & 11.82 \\
\hline Ganancia de talla & 8.39 & 6.13 \\
\hline
\end{tabular}

Los resultados obtenidos para la conversión y eficiencia alimenticia de ambos tratamientos del experimento, fueron mejor para concentrado comercial, donde se requiere $1.34 \mathrm{~kg}$ de alimento para obtener un kilo de carne, mientras que para el dieta elaborada se necesita $2.21 \mathrm{~kg}$, no así para el costo de alimento, ya que la dieta elaborada presenta $37 \%$ menos ( $\$ 7.3$ por $\mathrm{kg}$ ) que el concentrado comercial. Aun cuando la rentabilidad sobre los costos de alimentación son 119\% más en la dieta elaborada que en el concentrado comercial, los valores sobre la ganancia de peso, son mejores con el alimento comercial, por lo tanto el costo de $\mathrm{kg}$ de pescado producido es mayor. Pérez (2007), reporta que los costos de producción son determinantes esenciales, para la elección del alimento a suministrar en un sistema de producción de peces.

Los elementos de la dieta elaborada y utilizada en el presente trabajo, contienen algunos elementos de los empleados por Raa y Gildberg, 2002 y Shirai, 2006, quienes elaboraron dietas para alimentar tilapia, utilizando diferentes tipos de sustratos como fuente de carbohidratos en la producción de ensilajes, tales como, melaza de caña, avena, harina de maíz, yuca, trigo, arroz y además de almidón de yuca y maíz, los resultados fueron similares, el crecimiento fue más lento por la deficiencia de proteína en la dieta elaborada.

\section{CONCLUSIÓN}

En la acuacultura, la cantidad de proteína en el alimento para Tilapia tiene un efecto importante en el crecimiento, siendo los mejores resultados para el tratamiento con concentrado comercial. Partiendo de que la harina de pescado ha sido tradicionalmente la base de muchos alimentos comerciales para peces debido a su valor nutritivo y palatabilidad, por considerarse como el ingrediente que tiene el contenido más alto en calidad de proteínas, pero también es el más caro, la nutrición acuícola tiene ante sí el reto de implementar otras fuentes proteicas de origen vegetal con mayor disponibilidad y bajo costo.

\section{LITERATURA CITADA}

Auro, A.A., Fragoso, M.C., Ocampo, C.L. y Sumano, L. 2003. Estudio comparativo del crecimiento de Carpa Espejo (Cyprinus carpio var. specularis) alimentadas con cerdaza ensilada y empastillada, y con un alimento balanceado. REDVET En: http://www.veterinaria.org/revist as/redvet/n020203.html. consulta: 4 de mayo de 2015.

FAO. 2007. Problemáticas de la utilización de alimentos. Departamento de pesca y acuicultura.

En: http://www.fao.org/fi/website/FI 
RetrieveAction.do?dom=topic $\&$ fid $=28$

88. Consultado 7 de mayo de 2015

INEGI, 2010. Censo de Población y Vivienda

2010. Veracruz. Instituto Nacional de

Estadística Geografía e

Informática. México.

En:http://www.inegi.org.mx/sistemas/c

onsulta_resultados/iter2010.aspx ?c=27

$329 \&$ s=est (consultado el 28 de marzo de 2015).

Meyer C.G.J. 2007. Alimentación de la Tilapia del Nilo (Orechromis niloticus) con harina de fruta de Guanacaste (Enterolobium ciclocarpum). Tesis de Licenciatura de Ciencias y Producción Agropecuaria. Zamorano, Honduras. 20 pp.

Moreno, M.G., Hernández, J.G., Rovero, R., Tablante, A. y Rancel, L. 2000. Alimentación de Tilapia con raciones parciales de cáscaras de naranja. Ciencia y Tecnología Alimentaria; 3: 29-33.

https://doi.org/10.1080/11358120

Pérez G.O. 2007. Comparación de tres dietas para la producción de Oreochromis niloticus (Perciforme: cichlidae) bajo un sistema de producción intensivo. Tesis de Licenciatura. Universidad Veracruzana. Acayucan, Veracruz

Raa, J. y Gildberg, A. 2002. Fish silage: a review. En: Pilot scale lactic acid fermentation of shrimp wastes for chitin recovery. Process Biochemistry 37: 1359-1366.

https://doi.org/10.1016/S0032-9592(

Shirai, K. 2006. Situación actual y perspectivas pesqueros en la alimentación de del uso de ensilados de subproductos organismos acuáticos cultivados. En: Avances en Nutrición Acuícola VIII. VIII Simposium Internacional de Nutrición Acuícola. Noviembre 15-17. Monterrey. México.
Salas G. 2014,. Los productos y subproductos vegetales, animales y agroindustriales: Una alternativa para la alimentación de tilapia. Bio. Ciencias. 2014: 2-9 Bayamo, Cuba.

Souza. P., Tavares, L.G., Oliveira, J.S. y Ribeiro de Oliveira, S.J. 2012. Digestibility of agro-industrial byproducts in 200 and 300-g Nile tilapia. Revista Brasileira de Zootecnia 41: 462-466. https://doi.org/10.1590/S1516-359820 12000200032

USDA 2005. Proyecciones del Departamento de Agricultura de Estados Unidos.. Revista Claridades Agropecuarias SAGARPA 48-50. 
Copyright (c) 2016 Otoriel Hernández Porfirio, Santos Ángel Bruno Martinez, Guadalupe Castillo Capitán,

Maximino Zito Romero Figueroa y Maria Gisela V elázquez Silvestre

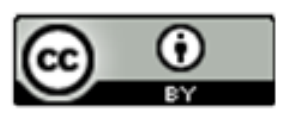

Este tex to está protegido por una licencia licencia Creative Commons 4.0 .

Usted es libre para Compartir —copiar y redistribuir el material en cualquier medio o form ato-y Adaptar el docum ento —remezclar, transformar y crear a partir del material- para cualquier propósito,, incluso para fines comerciales, siempre que cumpla la condición de:

Atribución: Usted debe dar crédito a la obra original de manera adecuada, proporcionar un enlace a la licencia, e in dicar si se han realizado cam bios. Puede hacerlo en cualquier form a razonable, pero no de form a tal que sugiera que tiene el apoyo del licenciante o 10 recibe por el uso que hace de la obra.

$\underline{\text { Resumencilicencia }}$ - Textocompletocilalicencia 